\title{
PARAMETRIC MARCINKIEWICZ INTEGRALS ON WEIGHTED HERZ SPACES
}

\author{
YUE HU AND YUESHAN WANG \\ Received 05 September, 2014
}

\begin{abstract}
Let $0<\rho<n$ and $\mu_{\Omega}^{\rho}$ be the parametric Marcinkiewicz integral. In this paper we shall obtain the strong type and weak type estimates of $\mu_{\Omega}^{\rho}$ on the weighted Herz spaces $\dot{K}_{q}^{\alpha, p}\left(\omega_{1}, \omega_{2}\right)$ with general weights. The boundedness of the commutators generated by $B M O$ functions and parametric Marcinkiewicz integral is also obtained.
\end{abstract}

2010 Mathematics Subject Classification: 42B25; 42B35

Keywords: parametric Marcinkiewicz integral, commutator, Muckenhoupt weight, BMO, Herz spaces, weak Herz space

\section{INTRODUCTION AND RESULTS}

Suppose that $\mathbb{S}^{n-1}$ is the unit sphere in $\mathbb{R}^{n}(n \geq 2)$ equipped with the normalized Lebesgue measure $d \sigma$. Let $\Omega$ be a homogeneous function of degree zero on $\mathbb{R}^{n}$ satisfying $\Omega \in L^{1}\left(\mathbb{S}^{n-1}\right)$ and

$$
\int_{\Phi^{n-1}} \Omega\left(x^{\prime}\right) d \sigma\left(x^{\prime}\right)=0,
$$

where $x^{\prime}=x /|x|$ for any $x \neq 0$. For $0<\rho<n$, Hörmander in [6] defined the parametric Marcinkiewicz integral operator $\mu_{\Omega}^{\rho}$ of higher dimension as follows.

$$
\mu_{\Omega}^{\rho}(f)(x)=\left(\int_{0}^{\infty}\left|F_{\Omega, t}^{\rho}(x)\right|^{2} \frac{d t}{t^{2 \rho+1}}\right)^{1 / 2}
$$

where

$$
F_{\Omega, t}^{\rho}(x)=\int_{|x-y| \leq t} \frac{\Omega(x-y)}{|x-y|^{n-\rho}} f(y) d y .
$$

The first author was supported in part by the National Natural Science Foundation of China, Grant No.11071057. 
Let $b$ be a locally integrable function, the commutator generated by parametric Marcinkiewcz integral $\mu_{\Omega}^{\rho}$ and $b$ is defined by

$$
\left[b, \mu_{\Omega}^{\rho}\right](f)(x)=\left(\int_{0}^{\infty}\left|\int_{|x-y| \leq t} \frac{\Omega(x-y)}{|x-y|^{n-\rho}}(b(x)-b(y)) f(y) d y\right|^{2} \frac{d t}{t^{2 \rho+1}}\right)^{\frac{1}{2}} .
$$

When $\rho=1$, we shall denote $\mu_{\Omega}^{1}$ simply by $\mu_{\Omega}$.

The area of the Marcinkiewcz integrals have been under intensive research. This operator $\mu_{\Omega}$ was first introduced by Stein in [15]. He proved that if $\Omega$

$\in \operatorname{Lip}_{\alpha}\left(\mathbb{S}^{n-1}\right)(0<\alpha \leq 1)$, then $\mu_{\Omega}$ is the operator of strong type $(p, p)$ for $1<p \leq 2$ and of weak type $(1,1)$. Here, we say that $\Omega \in L i p_{\alpha}\left(\mathbb{S}^{n-1}\right)$ if

$$
\left|\Omega\left(x^{\prime}\right)-\Omega\left(y^{\prime}\right)\right| \leq\left|x^{\prime}-y^{\prime}\right|^{\alpha}, \quad x^{\prime}, y^{\prime} \in \mathbb{S}^{n-1} .
$$

In 1990, Torchinsky and Wang in [16] considered the weighted case and proved that If $\Omega \in L i p_{\alpha}\left(\mathbb{S}^{n-1}\right)(0<\alpha \leq 1)$, and $b \in B M O\left(\mathbb{R}^{n}\right)$, then for all $1<p<\infty$, and $\omega \in A_{p}$ (Muckenhoupt weight class), $\mu_{\Omega}$ and $\left[b, \mu_{\Omega}\right]$ are all bounded in $L^{p}(\omega)$. On the other hand, in 1960, Hörmander [6] showed that if $\Omega \in L i p_{\alpha}\left(\mathbb{S}^{n-1}\right)(0<\alpha \leq 1)$, then for $0<\rho<n, \mu_{\Omega}^{\rho}$ is of strong type $(p, p)$ for all $1<p<\infty$. In [14] Shi and Jiang obtained the weighted $L^{p}$-boundedness of parametric Marcinkiewicz integral and its commutator.

Theorem A ([14]). Let $0<\rho<n$ and $\Omega \in L^{\infty}\left(\mathbb{S}^{n-1}\right)$. If $\omega \in A_{p}(1<p<\infty)$ and $b \in B M O\left(\mathbb{R}^{n}\right)$, then there exists a constant $C>0$ independent of $f$ such that

$$
\left\|\mu_{\Omega}^{\rho}(f)\right\|_{L^{p}(\omega)} \leq C\|f\|_{L^{p}(\omega)}
$$

and

$$
\left\|\left[b, \mu_{\Omega}^{\rho}\right](f)\right\|_{L^{p}(\omega)} \leq C\|b\|_{*}\|f\|_{L^{p}(\omega)} .
$$

Notice that $\operatorname{Lip} p_{\alpha}\left(\mathbb{S}^{n-1}\right)(0<\alpha \leq 1) \varsubsetneqq L^{\infty}\left(\mathbb{S}^{n-1}\right) \varsubsetneqq L^{1}\left(\mathbb{S}^{n-1}\right)$, then the results of Theorem A are extend and improve the results of Torchinsky and Wang in [16].

Let us recall the definition of Littlewood-Paley function

$$
S_{\Phi}(f)(x)=\left(\int_{\mathbb{R}^{n}}\left|\Phi_{t} * f(x)\right|^{2} \frac{d t}{t}\right)^{1 / 2},
$$

where $\Phi_{t}(x)=t^{-n} \Phi(x / t)$ and $\Phi \in L^{1}\left(\mathbb{R}^{n}\right)$ which satisfies $\int_{\mathbb{R}^{n}} \Phi(x) d x=0$. It is well known that the Littlewood-Paley function have long played important roles in harmonic analysis. When $\Phi$ is given by

$$
\Phi(x)=|x|^{-n+\rho} \Omega(x) \chi_{[0,1]}(|x|),
$$

where $\Omega$ be a homogeneous function of degree zero on $\mathbb{R}^{n}$ satisfying (1.1), then $\Phi(x)$ becomes the parametric Marcinkiewicz integral operator. Therefore, many authors have been interested in studying the boundedness properties of $\mu_{\Omega}^{\rho}$ on various function spaces, it can be seen in $[1-3,18]$. 
In [13], Lu, Yabuta and Yang obtained the boundedness results for sublinear operators on weighted Herz spaces with general Muckenhoupt weights. Recently, many authors considered the boundedness of operators on weighted Herz spaces with general Muckenhoupt weights. In [8], Komori and Matsuoka showed the boundedness of singular integral operators and fractional integrals on weighted Herz spaces. In [5], Guo and Jiang discussed the boundedness of commutators of singular integral operators on weighted Herz spaces, and as an application, they obtained the interior estimates on weighted Herz spaces for the solutions of some nondivergence elliptic equations. Hu, He and Wang [19] studied the boundedness of commutators of fractional integrals in generalized Herz spaces. More results concerning the boundedness of operators on Herz spaces can be seen in [12,17].

The main purpose of this paper is to consider the boundedness of parametric Marcinkiewicz integral on weighted Herz spaces with $A_{p}$ weights. At the extreme case, we will also prove that $\mu_{\Omega}^{\rho}$ is bounded from the weighted Herz spaces to the weighted weak Herz spaces. The boundedness of commutators generated by parametric Marcinkiewicz integral and $B M O$ functions on weighted Herz spaces is also considered.

Our main results in the paper are formulated as follows.

Theorem 1. Let $0<\rho<n$ and $\Omega \in L^{\infty}\left(\mathbb{S}^{n-1}\right)$. Suppose $0<p<\infty, 1<q<$ $\infty, \omega_{1} \in A_{q_{1}}$ and $\omega_{2} \in A_{q_{2}}$. Then $\mu_{\Omega}^{\rho}$ is bounded on $\dot{K}_{q}^{\alpha, p}\left(\omega_{1}, \omega_{2}\right)$ provided that $\omega_{1}$ and $\omega_{2}$ satisfy either of the following

(i) $\omega_{1}=\omega_{2}, 1 \leq q_{1}=q_{2} \leq q$ and $-n q_{1} / q<\alpha q_{1}<n\left(1-q_{1} / q\right)$;

(ii) $\omega_{1} \neq \omega_{2}, 1 \leq q_{1}<\infty, 1 \leq q_{2} \leq q$ and $0<\alpha q_{1}<n\left(1-q_{2} / q\right)$.

Theorem 2. Let $0<\rho<n$ and $\Omega \in L^{\infty}\left(\mathbb{S}^{n-1}\right)$. Suppose $0<p<1,1<q<$ $\infty, \omega_{1} \in A_{q_{1}}$ and $\omega_{2} \in A_{q_{2}}$. If $1 \leq q_{1}<\infty, 1 \leq q_{2} \leq q$ and $\alpha q_{1}=n\left(1-q_{2} / q\right)$, then $\mu_{\Omega}^{\rho}$ is bounded from $\dot{K}_{q}^{\alpha, p}\left(\omega_{1}, \omega_{2}\right)$ to $W \dot{K}_{q}^{\alpha, p}\left(\omega_{1}, \omega_{2}\right)$.

Theorem 3. Let $0<\rho<n$ and $\Omega \in L^{\infty}\left(\mathbb{S}^{n-1}\right)$. Suppose $1<q<\infty, \omega_{1} \in A_{q_{1}}$, $\omega_{2} \in A_{q_{2}}$ and $b \in B M O$. Then $\left[b, \mu_{\Omega}^{\rho}\right]$ is bounded on $\dot{K}_{q}^{\alpha, p}\left(\omega_{1}, \omega_{2}\right)$ provided that $\omega_{1}$ and $\omega_{2}$ satisfy either of the following

(i) $\omega_{1}=\omega_{2}, 1 \leq q_{1}=q_{2} \leq q$ and $-n q_{1} / q<\alpha q_{1}<n\left(1-q_{1} / q\right)$;

(ii) $\omega_{1} \neq \omega_{2}, 1 \leq q_{1}<\infty, 1 \leq q_{2} \leq q$ and $0<\alpha q_{1}<n\left(1-q_{2} / q\right)$.

Throughout this paper, unless otherwise indicated, $C$ will be used to denote a positive constant that is not necessarily the same at each occurrence.

\section{DEFINITIONS AND PRELIMINARIES}

We begin this section with some properties of $A_{p}$ weights which play important role in the proofs of our main results.

A weight $\omega$ is a nonnegative, locally integrable function on $\mathbb{R}^{n}$. Let $B=B\left(x_{0}, r\right)$ denote the ball with the center $x_{0}$ and radius $r$, and let $\lambda B=B\left(x_{0}, \lambda r\right)$ for any $\lambda>0$. 
For a given weight function $\omega$ and a measurable set $E$, we also denote the Lebesgue measure of $E$ by $|E|$ and set weighted measure $\omega(E)=\int_{E} \omega(x) d x$.

Definition 1. A weight $\omega$ is said to belong to $A_{p}$ for $1<p<\infty$, if there exists a constant $C$ such that for every ball $B \subset \mathbb{R}^{n}$,

$$
\left(\frac{1}{|B|} \int_{B} \omega(x) d x\right)\left(\frac{1}{|B|} \int_{B} \omega(x)^{1-p^{\prime}} d x\right)^{p-1} \leq C,
$$

where $s^{\prime}$ is the dual of $s$ such that $1 / s+1 / s^{\prime}=1$.

The class $A_{1}$ is defined by replacing the above inequality with

$$
\frac{1}{|B|} \int_{B} \omega(y) d y \leq C \cdot \underset{x \in B}{\operatorname{essinf}} w(x) .
$$

By (2.1), we have

$$
\left(\int_{B} \omega(x) d x\right)\left(\int_{B} \omega(x)^{1-p^{\prime}} d x\right)^{p-1} \leq C|B|^{p} .
$$

The classical $A_{p}$ weight theory was first introduced by Muckenhoupt in the study of weighted $L^{p}$-boundedness of Hardy-Littlewood maximal function in [10].

Lemma $1([4,10])$. Let $1 \leq p<\infty$ and $\omega \in A_{p}$. Then the following statements are true:

(i) There exists constant $C$ such that

$$
\frac{\omega\left(B_{k}\right)}{\omega\left(B_{j}\right)} \leq C 2^{n p(k-j)} \text { for } k>j ;
$$

(ii) For any $1<p<\infty$, there exists $1<q<p$ such that $\omega \in A_{q}\left(\mathbb{R}^{n}\right)$;

(iii) There exist two constants $C$ and $\delta>0$ such that for any measurable set $E \subset$ $B$,

$$
\frac{\omega(E)}{\omega(B)} \leq C\left(\frac{|E|}{|B|}\right)^{\delta}
$$

(iv) For any $1<p<\infty$, one has $\omega^{1-p^{\prime}} \in A_{p^{\prime}}\left(\mathbb{R}^{n}\right)$.

If $\omega$ satisfies (2.4), we say $\omega \in A_{\infty}\left(\mathbb{R}^{n}\right)$. It is well known that

$$
A_{\infty}\left(\mathbb{R}^{n}\right)=\bigcup_{1 \leq p<\infty} A_{p}\left(\mathbb{R}^{n}\right) .
$$

Let $B_{k}=B\left(0,2^{k}\right)=\left\{x \in \mathbb{R}^{n}:|x| \leq 2^{k}\right\}$ and $C_{k}=B_{k} \backslash B_{k-1}$ for any $k \in \mathbb{Z}$. Denote $\chi_{k}=\chi_{C_{k}}$ for $k \in \mathbb{Z}$, where $\chi_{E}$ is the characteristic function of the set $E$. In [9], Lu and Yang introduced weighted Herz spaces. 
Definition 2. Let $\alpha \in \mathbb{R}, 0<p, q<\infty$ and $\omega_{1}, \omega_{2}$ be two weight functions on $\mathbb{R}^{n}$. The homogenous weighted Herz space $\dot{K}_{q}^{\alpha, p}\left(\omega_{1}, \omega_{2}\right)$ is defined by

$$
\dot{K}_{q}^{\alpha, p}\left(\omega_{1}, \omega_{2}\right)=\left\{f \in L_{l o c}^{q}\left(\mathbb{R}^{n} \backslash\{0\}, \omega_{2}\right):\|f\|_{\dot{K}_{q}^{\alpha, p}\left(\omega_{1}, \omega_{2}\right)}<\infty\right\},
$$

where

$$
\|f\|_{\dot{K}_{q}^{\alpha, p}\left(\omega_{1}, \omega_{2}\right)}=\left(\sum_{k=-\infty}^{\infty} \omega_{1}\left(B_{k}\right)^{\alpha p / n}\left\|f \chi_{k}\right\|_{L^{q}\left(\omega_{2}\right)}^{p}\right)^{1 / p} .
$$

For any $k \in \mathbb{Z}, \lambda>0$ and any measurable function $f$ on $\mathbb{R}^{n}$, we write $E_{k}(\lambda, f)=$ $\left\{x \in C_{k}:|f(x)|>\lambda\right\}$.

Definition 3 ([13]). Let $\alpha \in \mathbb{R}, 0<p, q<\infty$ and $\omega_{1}, \omega_{2}$ be two weight function on $\mathbb{R}^{n}$. A measurable function $f$ on $\mathbb{R}^{n}$ is said to belong to the homogeneous weighted weak Herz space $W \dot{K}_{q}^{\alpha, p}\left(\omega_{1}, \omega_{2}\right)$ if

$$
\|f\|_{W \dot{K}_{q}^{\alpha, p}\left(\omega_{1}, \omega_{2}\right)}=\sup _{\lambda>0} \lambda\left(\sum_{k=-\infty}^{\infty} \omega_{1}\left(B_{k}\right)^{\alpha p / n}\left[\omega_{2}\left(E_{k}(\lambda, f)\right)\right]^{p / q}\right)^{1 / p}<\infty .
$$

Obviously, if $\alpha=0$, then $\dot{K}_{p}^{0, p}\left(\omega_{1}, \omega_{2}\right)=L^{p}\left(\omega_{2}\right)$ and $W \dot{K}_{p}^{0, p}\left(\omega_{1}, \omega_{2}\right)$ $=W L^{p}\left(\omega_{2}\right)$ for any $0<p<\infty$. Thus, weighted Herz spaces are generalizations of the weighted Lebesgue spaces.

Definition 4. A locally integrable function $b$ is said to be in $B M O\left(\mathbb{R}^{n}\right)$ if

$$
\sup _{B} \frac{1}{|B|} \int_{B}\left|b(x)-b_{B}\right| d x=\|b\|_{*}<\infty,
$$

where $b_{B}=\frac{1}{|B|} \int_{B} b(y) d y$.

Lemma 2. (John-Nirenberg inequality, see [7]) Let $b \in B M O\left(\mathbb{R}^{n}\right)$. Then for any ball $B \subset \mathbb{R}^{n}$, there exist constant $C_{1}, C_{2}$ such that for all $\lambda>0$,

$$
\left|\left\{x \in B:\left|b(x)-b_{B}\right|>\lambda\right\}\right| \leq C_{1}|B| \exp \left(-C_{2} \lambda /\|b\|_{*}\right) .
$$

Lemma 3 ([11]). Let $\omega \in A_{\infty}$. Then the norm of $B M O\left(\omega, \mathbb{R}^{n}\right)$ is equivalent to the norm of $B M O\left(\mathbb{R}^{n}\right)$, where

$$
B M O\left(\omega, \mathbb{R}^{n}\right)=\left\{b:\|b\|_{*, \omega}=\sup _{B \subset \mathbb{R}^{n}} \frac{1}{\omega(B)} \int_{B}\left|b(x)-b_{B, \omega}\right| \omega(x) d x\right\},
$$

and

$$
b_{B, \omega}=\frac{1}{\omega(B)} \int_{B} b(z) \omega(z) d z .
$$

Lemma 4. Suppose $\omega \in A_{\infty}\left(\mathbb{R}^{n}\right), b \in B M O\left(\mathbb{R}^{n}\right)$. Then for any $p \geq 1$ we have

$$
\left(\frac{1}{\omega(B)} \int_{B}\left|b(x)-b_{B, \omega}\right|^{p} \omega(x) d x\right)^{1 / p} \leq C\|b\|_{*} .
$$


Proof. Since $\omega(x) \in A_{\infty}\left(\mathbb{R}^{n}\right)$, then (iii) of Lemma 1 and Lemma 2 imply

$$
\omega\left(\left\{x \in B:\left|b(x)-b_{B}\right|>\lambda\right\}\right) \leq C \omega(B) \exp \left(-C_{2} \delta \lambda /\|b\|_{*}\right) .
$$

So

$$
\begin{aligned}
\int_{B}\left|b(x)-b_{B}\right|^{p} \omega(x) d x & \leq \int_{0}^{\infty} p \lambda^{p-1} \omega\left(\left\{x \in B:\left|b(x)-b_{B}\right|>\lambda\right\}\right) d \lambda \\
& \leq C \omega(B) \int_{0}^{\infty} p \lambda^{p-1} \exp \left(-C_{2} \delta \lambda\|b\|_{*}\right) d \lambda \\
& \leq C \omega(B)\|b\|_{*}^{p} .
\end{aligned}
$$

Thus

$$
\frac{1}{\omega(B)} \int_{B}\left|b(x)-b_{B, \omega}\right|^{p} \omega(x) d x \leq \frac{C}{\omega(B)} \int_{B}\left|b(x)-b_{B}\right|^{p} \omega(x) d x \leq C\|b\|_{*}^{p} .
$$

\section{Proof of Theorem 1}

Let $f \in \dot{K}_{q}^{\alpha, p}\left(\omega_{1}, \omega_{2}\right)$. Then

$$
\begin{aligned}
& \left\|\mu_{\Omega}^{\rho}(f)\right\|_{\dot{K}_{q}^{\alpha, p}\left(\omega_{1}, \omega_{2}\right)}^{p} \\
\leq & C \sum_{j=-\infty}^{\infty}\left(\omega_{1}\left(B_{j}\right)\right)^{\frac{\alpha p}{n}}\left(\sum_{k=-\infty}^{j-2} \|\left(\mu_{\Omega}^{\rho}\left(f \chi_{k}\right) \chi_{j}(x) \|_{L^{q}\left(\omega_{2}\right)}\right)^{p}\right. \\
& +C \sum_{j=-\infty}^{\infty}\left(\omega_{1}\left(B_{j}\right)\right)^{\frac{\alpha p}{n}}\left(\sum_{k=j-1}^{j+1} \|\left(\mu_{\Omega}^{\rho}\left(f \chi_{k}\right) \chi_{j}(x) \|_{L^{q}\left(\omega_{2}\right)}\right)^{p}\right. \\
& +C \sum_{j=-\infty}^{\infty}\left(\omega_{1}\left(B_{j}\right)\right)^{\frac{\alpha p}{n}}\left(\sum_{k=j+2}^{\infty} \|\left(\mu_{\Omega}^{\rho}\left(f \chi_{k}\right) \chi_{j}(x) \|_{L^{q}\left(\omega_{2}\right)}\right)^{p}\right. \\
= & E_{1}+E_{2}+E_{3} .
\end{aligned}
$$

By the fact that $\mu_{\Omega}^{\rho}$ is a bounded operator on $L^{q}\left(\omega_{2}\right)$, we get

$$
\begin{aligned}
E_{2} & =C \sum_{j=-\infty}^{\infty}\left(\omega_{1}\left(B_{j}\right)\right)^{\frac{\alpha p}{n}}\left(\sum_{k=j-1}^{j+1} \|\left(\mu_{\Omega}^{\rho}\left(f \chi_{k}\right) \chi_{j}(x) \|_{L^{q}\left(\omega_{2}\right)}\right)^{p}\right. \\
& \leq C \sum_{j=-\infty}^{\infty}\left(\omega_{1}\left(B_{j}\right)\right)^{\frac{\alpha p}{n}}\left(\sum_{k=j-1}^{j+1}\left\|f \chi_{k}\right\|_{L^{q}\left(\omega_{2}\right)}\right)^{p} \\
& \leq C\|f\|_{\dot{K}_{q}^{\alpha, p}\left(\omega_{1}, \omega_{2}\right)}^{p} .
\end{aligned}
$$


For any $x \in C_{j}$ and $y \in C_{k} \cap\{y:|x-y|<t\}$ with $j \geq k+2$, we have $t>|x-y| \geq$ $|x|-|y| \geq|x| / 2 \geq C 2^{j n}$. So

$$
\begin{aligned}
\left|\mu_{\Omega}^{\rho}\left(f \chi_{k}\right) \chi_{j}(x)\right| & =\left(\int_{0}^{\infty}\left|\int_{|x-y| \leq t} \frac{\Omega(x-y)}{|x-y|^{n-\rho}} f_{k}(y) d y\right|^{2} \frac{d t}{t^{2 \rho+1}}\right)^{1 / 2} \\
& \leq C 2^{-j(n-\rho)}\left\|f \chi_{k}\right\|_{L^{1}}\left(\int_{C 2^{j n}}^{\infty} \frac{d t}{t^{2 \rho+1}}\right)^{1 / 2} .
\end{aligned}
$$

Then

$$
\left|\mu_{\Omega}^{\rho}\left(f \chi_{k}\right) \chi_{j}(x)\right|=C 2^{-j n}\left\|f \chi_{k}\right\|_{L^{1}},
$$

and

$$
\left\|\mu_{\Omega}^{\rho}\left(f \chi_{k}\right) \chi_{j}(x)\right\|_{L^{q}\left(\omega_{2}\right)} \leq C 2^{-j n} \omega_{2}\left(B_{j}\right)^{1 / q}\left\|f \chi_{k}\right\|_{L^{1}} .
$$

By Hölder's inequality,

$$
\left\|f \chi_{k}\right\|_{L^{1}} \leq C\left\|f \chi_{k}\right\|_{L^{q}\left(\omega_{2}\right)}\left(\int_{B_{k}} \omega_{2}(x)^{1-q^{\prime}} d x\right)^{1 / q^{\prime}} .
$$

Since $\omega_{2} \in A_{q_{2}}\left(\mathbb{R}^{n}\right) \subset A_{q}\left(\mathbb{R}^{n}\right)$, by (2.2) and (2.3) we get,

$$
\begin{aligned}
& \left(\int_{B_{k}} \omega_{2}(x)^{1-q^{\prime}} d x\right)^{1 / q^{\prime}}\left(\int_{B_{j}} \omega_{2}(x) d x\right)^{1 / q} \\
= & \left(\int_{B_{k}} \omega_{2}(x)^{1-q^{\prime}} d x\right)^{1 / q^{\prime}}\left(\int_{B_{k}} \omega_{2}(x) d x\right)^{1 / q}\left(\frac{\omega_{2}\left(B_{j}\right)}{\omega_{2}\left(B_{k}\right)}\right)^{1 / q} \\
\leq & C 2^{k n+(j-k) n q_{2} / q} .
\end{aligned}
$$

Then, for $j \geq k+2$ we have

$$
\left\|\mu_{\Omega}^{\rho}\left(f \chi_{k}\right) \chi_{j}(x)\right\|_{L^{q}\left(\omega_{2}\right)} \leq C\left\|f \chi_{k}\right\|_{L^{q}\left(\omega_{2}\right)} 2^{(j-k) n\left(q_{2} / q-1\right)} .
$$

Thus

$$
\begin{aligned}
E_{1} & =C \sum_{j=-\infty}^{\infty}\left(\omega_{1}\left(B_{j}\right)\right)^{\frac{\alpha p}{n}}\left(\sum_{k=-\infty}^{j-2}\left\|\mu_{\Omega}^{\rho}\left(f \chi_{k}\right) \chi_{j}(x)\right\|_{L^{q}\left(\omega_{2}\right)}\right)^{p} \\
& \leq C\|b\|_{*}^{p} \sum_{j=-\infty}^{\infty}\left(\sum_{k=-\infty}^{j-2}\left(\omega_{1}\left(B_{j}\right)\right)^{\frac{\alpha}{n}}\left\|f \chi_{k}\right\|_{L^{q}\left(\omega_{2}\right)} 2^{(j-k) n\left(q_{2} / q-1\right)}\right)^{p} \\
& \leq C\|b\|_{*}^{p} \sum_{j=-\infty}^{\infty}\left(\sum_{k=-\infty}^{j-2}\left(\omega_{1}\left(B_{k}\right)\right)^{\frac{\alpha}{n}}\left\|f \chi_{k}\right\|_{L^{q}\left(\omega_{2}\right)} 2^{(j-k)\left(\alpha q_{1}+q_{2} n / q-n\right)}\right)^{p} .
\end{aligned}
$$


When $0<p \leq 1$, we get

$$
\begin{aligned}
E_{1} & \leq C\|b\|_{*}^{p} \sum_{k=-\infty}^{\infty}\left(\omega_{1}\left(B_{k}\right)\right)^{\frac{\alpha p}{n}}\left\|f \chi_{k}\right\|_{L^{q}\left(\omega_{2}\right)}^{p} \sum_{j=-\infty}^{k+2} 2^{(j-k) p\left(\alpha q_{1}+q_{2} n / q-n\right)} \\
& \leq C\|b\|_{*}^{p} \sum_{k=-\infty}^{\infty}\left(\omega_{1}\left(B_{k}\right)\right)^{\frac{\alpha p}{n}}\left\|f \chi_{k}\right\|_{L^{q}\left(\omega_{2}\right)}^{p} \\
& \leq C\|b\|_{*}^{p}\|f\|_{\dot{K}_{q}^{\alpha, p}\left(\omega_{1}, \omega_{2}\right)\left(\mathbb{R}^{n}\right)}^{p} .
\end{aligned}
$$

When $p>1$, by Hölder's inequality we get

$$
\begin{aligned}
E_{1} \leq & C\|b\|_{*}^{p} \sum_{j=-\infty}^{\infty}\left(\sum_{k=-\infty}^{j-2}\left(\omega_{1}\left(B_{k}\right)\right)^{\frac{\alpha p}{n}}\left\|f \chi_{k}\right\|_{L^{q}\left(\omega_{2}\right)}^{p} 2^{(j-k)\left(\alpha q_{1}+q_{2} n / q-n\right)}\right) \\
& \left(\sum_{k=-\infty}^{j-2} 2^{(j-k)\left(\alpha q_{1}+q_{2} n / q-n\right)}\right)^{p / p^{\prime}} \\
\leq & C\|b\|_{*}^{p} \sum_{k=-\infty}^{\infty}\left(\omega_{1}\left(B_{k}\right)\right)^{\frac{\alpha p}{n}}\left\|f \chi_{k}\right\|_{L^{q}\left(\omega_{2}\right)}^{p} \\
\leq & C\|b\|_{*}^{p}\|f\|_{\dot{K}_{q}^{\alpha, p}\left(\omega_{1}, \omega_{2}\right)\left(\mathbb{R}^{n}\right)^{p}}^{p}
\end{aligned}
$$

Let us now turn to estimate the last term $E_{3}$. In the case $k \geq j+2$, for any $x \in C_{j}$ and $y \in C_{k} \cap\{y:|x-y|<t\}$, we have $t>|x-y| \geq|y|-|x| \geq|y| / 2 \geq C 2^{k n}$. Then, similar to the estimates of (3.1),

$$
\left|\mu_{\Omega}^{\rho}\left(f \chi_{k}\right) \chi_{j}(x)\right| \leq C 2^{-k n}\left\|f \chi_{k}\right\|_{L^{1}} .
$$

So,

$$
\left\|\mu_{\Omega}^{\rho}\left(f \chi_{k}\right) \chi_{j}(x)\right\|_{L^{q}\left(\omega_{2}\right)} \leq C 2^{-k n} \omega_{2}\left(B_{j}\right)^{1 / q}\left\|f \chi_{k}\right\|_{L^{1}} .
$$

By (2.2) and (2.4),

$$
\left(\int_{B_{k}} \omega_{2}(x)^{1-q^{\prime}} d x\right)^{1 / q^{\prime}}\left(\int_{B_{j}} \omega_{2}(x) d x\right)^{1 / q} \leq C 2^{k n+(j-k) \delta_{2} n / q} .
$$

Combining with (3.2), (3.5) and (3.6), we have

$$
\left\|\mu_{\Omega}^{\rho}\left(f \chi_{k}\right) \chi_{j}(x)\right\|_{L^{q}\left(\omega_{2}\right)} \leq C\left\|f \chi_{k}\right\|_{L^{q}\left(\omega_{2}\right)} 2^{(j-k) \delta_{2} n / q}
$$

for $j \leq k-2$. 
When $0<p \leq 1$, we get

$$
\begin{aligned}
E_{3} & \leq C\|b\|_{*}^{p} \sum_{j=-\infty}^{\infty} \omega_{1}\left(B_{j}\right)^{\frac{\alpha p}{n}} \sum_{k=j+2}^{\infty}\left\|f \chi_{k}\right\|_{L^{q}\left(\omega_{2}\right)}^{p} 2^{(j-k) p \delta_{2} n / q} \\
& \leq C\|b\|_{*}^{p} \sum_{k=-\infty}^{\infty}\left(\omega_{1}\left(B_{k}\right)\right)^{\frac{\alpha p}{n}}\left\|f \chi_{k}\right\|_{L^{q}\left(\omega_{2}\right)}^{p} \sum_{j=-\infty}^{k-2}\left(\frac{\omega_{1}\left(B_{j}\right)}{\omega_{1}\left(B_{k}\right)}\right)^{\frac{\alpha p}{n}} 2^{(j-k) p \delta_{2} n / q} \\
& \leq C\|b\|_{*}^{p} \sum_{k=-\infty}^{\infty}\left(\omega_{1}\left(B_{k}\right)\right)^{\frac{\alpha p}{n}}\left\|f \chi_{k}\right\|_{L^{q}\left(\omega_{2}\right)}^{p} \sum_{j=-\infty}^{k-2} 2^{(j-k) p\left(\delta_{1} \alpha+\delta_{2} n / q\right)} \\
& \leq C\|b\|_{*}^{p}\|f\|_{\dot{K}_{q}^{\alpha, p}\left(\omega_{1}, \omega_{2}\right)}^{p} .
\end{aligned}
$$

When $q>1$, by Hölder's inequality we get

$$
\begin{aligned}
E_{3} & \left.\leq C\|b\|_{*}^{p} \sum_{j=-\infty}^{\infty}\left(\sum_{k=j+2}^{\infty}\left(\omega_{1}\left(B_{k}\right)\right)^{\frac{\alpha}{n}}\left\|f \chi_{k}\right\|_{L^{q}\left(\omega_{2}\right)}\right)^{(j-k)\left(\delta_{1} \alpha+\delta_{2} n / q\right)}\right)^{p} \\
& \leq C\|b\|_{*}^{p} \sum_{j=-\infty}^{\infty}\left(\sum_{k=j+2}^{\infty}\left(\omega_{1}\left(B_{k}\right)\right)^{\frac{\alpha p}{n}}\left\|f \chi_{k}\right\|_{L^{q}\left(\omega_{2}\right)}^{p} 2^{(j-k)\left(\delta_{1} \alpha+\delta_{2} n / q\right)}\right) \\
& \left(\sum_{k=j+2}^{\infty} 2^{(j-k)\left(\delta_{1} \alpha+\delta_{2} n / q\right)}\right)^{p / p^{\prime}} \\
& \leq C\|b\|_{*}^{p} \sum_{k=-\infty}^{\infty}\left(\omega_{1}\left(B_{k}\right)\right)^{\frac{\alpha p}{n}}\left\|f \chi_{k}\right\|_{L^{q}\left(\omega_{2}\right)}^{p} \sum_{j=-\infty}^{k-2} 2^{(j-k)\left(\delta_{1} \alpha+\delta_{2} n / q\right)} \\
& \leq C\|b\|_{*}^{p}\|f\|_{\dot{K}_{q}^{\alpha, p}\left(\omega_{1}, \omega_{2}\right)}^{p} .
\end{aligned}
$$

Combining the above estimates for $E_{1}, E_{2}$ and $E_{3}$, the proof of Theorem 1 is completed.

\section{Proof of THEOREM 2}

Let $f \in \dot{K}_{q}^{\alpha, p}\left(\omega_{1}, \omega_{2}\right)$. Then

$$
\begin{aligned}
& \lambda^{p} \sum_{j=-\infty}^{\infty}\left(\omega_{1}\left(B_{j}\right)\right)^{\frac{\alpha p}{n}}\left(\omega_{2}\left(\left\{x \in C_{j}:\left|\mu_{\Omega}^{\rho}(f)(x)\right|>\lambda\right\}\right)\right)^{p / q} \\
\leq & \lambda^{p} \sum_{j=-\infty}^{\infty}\left(\omega_{1}\left(B_{j}\right)\right)^{\frac{\alpha p}{n}}\left(\omega_{2}\left(\left\{x \in C_{j}: \sum_{k=-\infty}^{j-2}\left|\mu_{\Omega}^{\rho}\left(f_{k}\right)(x)\right|>\lambda / 3\right\}\right)\right)^{p / q}
\end{aligned}
$$




$$
\begin{aligned}
& +\lambda^{p} \sum_{j=-\infty}^{\infty}\left(\omega_{1}\left(B_{j}\right)\right)^{\frac{\alpha p}{n}}\left(\omega_{2}\left(\left\{x \in C_{j}: \sum_{k=j-1}^{j+1}\left|\mu_{\Omega}^{\rho}\left(f_{k}\right)(x)\right|>\lambda / 3\right\}\right)\right)^{p / q} \\
& +\lambda^{p} \sum_{j=-\infty}^{\infty}\left(\omega_{1}\left(B_{j}\right)\right)^{\frac{\alpha p}{n}}\left(\omega_{2}\left(\left\{x \in C_{j}: \sum_{k=j-2}^{\infty}\left|\mu_{\Omega}^{\rho}\left(f_{k}\right)(x)\right|>\lambda / 3\right\}\right)\right)^{p / q} \\
& =F_{1}+F_{2}+F_{3} .
\end{aligned}
$$

Applying Chebyshev's inequality [4] and Theorem A, we obtain

$$
\begin{aligned}
F_{2} & \leq C \lambda^{p} \sum_{j=-\infty}^{\infty}\left(\omega_{1}\left(B_{j}\right)\right)^{\frac{\alpha p}{n}}\left(\frac{1}{\lambda^{q}} \sum_{k=j-1}^{j+1}\left\|\mu_{\Omega}^{\rho}\left(f_{k}\right)\right\|_{L^{q}\left(\omega_{2}\right)}^{q}\right)^{p / q} \\
& \leq C \sum_{j=-\infty}^{\infty}\left(\omega_{1}\left(B_{j}\right)\right)^{\frac{\alpha p}{n}}\left(\sum_{k=j-1}^{j+1}\left\|f \chi_{k}\right\|_{L^{q}\left(\omega_{2}\right)}\right)^{p} \\
& \leq C\|f\|_{\dot{K}_{q}^{\alpha, p}\left(\omega_{1}, \omega_{2}\right)}^{p} .
\end{aligned}
$$

For any $x \in C_{j}$ and $y \in C_{k} \cap\{y:|x-y|<t\}$ with $j \geq k+2$, by the inequalities (3.1), (3.2) and (3.3) we have

$$
\left|\mu_{\Omega}^{\rho}\left(f \chi_{k}\right) \chi_{j}(x)\right| \leq C\left(\omega_{2}\left(B_{j}\right)\right)^{-1 / q} 2^{-j n+k n+(j-k) n q_{2} / q}\left\|f \chi_{k}\right\|_{L^{q}\left(\omega_{2}\right)} .
$$

Noting the fact $\alpha q_{1}=n\left(1-q_{2} / q\right)$, then

$$
\left|\mu_{\Omega}^{\rho}\left(f \chi_{k}\right) \chi_{j}(x)\right| \leq C\left(\omega_{2}\left(B_{j}\right)\right)^{-1 / q} 2^{(k-j) \alpha q_{1}}\left\|f \chi_{k}\right\|_{L^{q}\left(\omega_{2}\right)} .
$$

Moreover, since $0<p \leq 1$, then for any $x \in C_{j}$,

$$
\begin{aligned}
& \sum_{k=-\infty}^{j-2}\left|\mu_{\Omega}^{\rho}\left(f_{k}\right)(x)\right| \\
\leq & C\left(\omega_{2}\left(B_{j}\right)\right)^{-1 / q}\left(\omega_{1}\left(B_{j}\right)\right)^{-\alpha / n} \\
& \sum_{k=-\infty}^{j-2} 2^{(k-j) \alpha q_{1}}\left(\omega_{1}\left(B_{k}\right)\right)^{\frac{\alpha}{n}}\left\|f \chi_{k}\right\|_{L^{q}\left(\omega_{2}\right)}\left(\frac{\omega_{1}\left(B_{j}\right)}{\omega_{1}\left(B_{k}\right)}\right)^{\frac{\alpha}{n}} \\
\leq & C\left(\omega_{2}\left(B_{j}\right)\right)^{-1 / q}\left(\omega_{1}\left(B_{j}\right)\right)^{-\alpha / n} \sum_{k=-\infty}^{j-2}\left(\omega_{1}\left(B_{k}\right)\right)^{\frac{\alpha}{n}}\left\|f \chi_{k}\right\|_{L^{q}\left(\omega_{2}\right)}
\end{aligned}
$$




$$
\begin{aligned}
& \leq C\left(\omega_{2}\left(B_{j}\right)\right)^{-1 / q}\left(\omega_{1}\left(B_{j}\right)\right)^{-\alpha / n}\left(\sum_{k=-\infty}^{j-2}\left(\omega_{1}\left(B_{k}\right)\right)^{\frac{\alpha p}{n}}\left\|f \chi_{k}\right\|_{L^{q}\left(\omega_{2}\right)}^{p}\right)^{1 / p} \\
& \leq C\left(\omega_{2}\left(B_{j}\right)\right)^{-1 / q}\left(\omega_{1}\left(B_{j}\right)\right)^{-\alpha / n}\|f\|_{\dot{K}_{q}^{\alpha, p}\left(\omega_{1}, \omega_{2}\right)} .
\end{aligned}
$$

If

$$
\left\{x \in C_{j}: \sum_{k=-\infty}^{j-2}\left|\mu_{\Omega}^{\rho}\left(f_{k}\right)(x)\right|>\lambda / 3\right\}=\varnothing,
$$

then

$$
F_{1} \leq C\|f\|_{\dot{K}_{p}^{\alpha, p}\left(\omega_{1}, \omega_{2}\right)}^{p}
$$

holds is trivially. Now we suppose

$$
\left\{x \in C_{j}: \sum_{k=-\infty}^{j-2}\left|\mu_{\Omega}^{\rho}\left(f_{k}\right)(x)\right|>\lambda / 3\right\} \neq \varnothing .
$$

Let

$$
S_{j}=\left(\omega_{2}\left(B_{j}\right)\right)^{-1 / q}\left(\omega_{1}\left(B_{j}\right)\right)^{-\alpha / n} .
$$

Since $\alpha>0$, it is easy to see that

$$
\lim _{j \rightarrow \infty} S_{j}=0 .
$$

Then for any $\lambda>0$, we can find a maximal positive integer $j_{\lambda}$ such that

$$
\lambda / 3 \leq C S_{j_{\lambda}}\|f\|_{\dot{K}_{q}^{\alpha, p}\left(\omega_{1}, \omega_{2}\right)} .
$$

So

$$
\begin{aligned}
F_{1} & \leq \lambda^{p} \sum_{j=-\infty}^{j_{\lambda}}\left(\omega_{2}\left(B_{j}\right)\right)^{p / q}\left(\omega_{1}\left(B_{j}\right)\right)^{\alpha p / n} \\
& \leq C\|f\|_{\dot{K}_{p}^{\alpha, p}\left(\omega_{1}, \omega_{2}\right)}^{p} \sum_{j=-\infty}^{j_{\lambda}}\left(\frac{\omega_{1}\left(B_{j}\right)}{\omega_{1}\left(B_{j_{\lambda}}\right)}\right)^{\frac{\alpha p}{n}}\left(\frac{\omega_{2}\left(B_{j}\right)}{\omega_{2}\left(B_{j_{\lambda}}\right)}\right)^{\frac{p}{q}} \\
& \leq C\|f\|_{\dot{K}_{p}^{\alpha, p}\left(\omega_{1}, \omega_{2}\right)}^{p} \sum_{j=-\infty}^{j_{\lambda}} 2^{\left(j-j_{\lambda}\right)\left(\delta_{1} \alpha p+\delta_{2} p n / q\right)} \\
& \leq C\|f\|_{\dot{K}_{q}^{\alpha, p}\left(\omega_{1}, \omega_{2}\right)}^{p}
\end{aligned}
$$

Let us now estimate $F_{3}$. From (3.2), (3.4) and (3.6) we have

$$
\left|\mu_{\Omega}^{\rho}\left(f \chi_{k}\right) \chi_{j}(x)\right| \leq C\left(\omega_{2}\left(B_{j}\right)\right)^{-1 / q} 2^{(j-k) \delta_{2} n / q}\left\|f \chi_{k}\right\|_{L^{q}\left(\omega_{2}\right)}
$$


for $j \geq k-2$. So

$$
\begin{aligned}
& \sum_{k=j-2}^{\infty}\left|\mu_{\Omega}^{\rho}\left(f_{k}\right)(x)\right| \\
& \leq C\left(\omega_{2}\left(B_{j}\right)\right)^{-1 / q}\left(\omega_{1}\left(B_{j}\right)\right)^{-\alpha / n} \\
& \sum_{k=j-2}^{\infty}\left(\omega_{1}\left(B_{k}\right)\right)^{\alpha / n}\left\|f \chi_{k}\right\|_{L^{q}\left(\omega_{2}\right)} 2^{(j-k) \delta_{2} n / q}\left(\frac{\omega_{1}\left(B_{j}\right)}{\omega_{1}\left(B_{k}\right)}\right)^{\alpha / n} \\
& \leq C\left(\omega_{2}\left(B_{j}\right)\right)^{-1 / q}\left(\omega_{1}\left(B_{j}\right)\right)^{-\alpha / n} \sum_{k=j-2}^{\infty}\left(\omega_{1}\left(B_{k}\right)\right)^{\alpha / n}\left\|f \chi_{k}\right\|_{L^{q}} 2^{(j-k)\left(\delta_{2} n / q+\alpha \delta_{1}\right)} \\
& \leq C\left(\omega_{2}\left(B_{j}\right)\right)^{-1 / q}\left(\omega_{1}\left(B_{j}\right)\right)^{-\alpha / n} \sum_{k=j-2}^{\infty}\left(\omega_{1}\left(B_{k}\right)\right)^{\alpha / n}\left\|f \chi_{k}\right\|_{L^{q}}
\end{aligned}
$$

Noting that $0<p \leq 1$, we have

$$
\begin{aligned}
& \sum_{k=j-2}^{\infty}\left|\mu_{\Omega}^{\rho}\left(f_{k}\right)(x)\right| \\
\leq & C\left(\omega_{2}\left(B_{j}\right)\right)^{-1 / q}\left(\omega_{1}\left(B_{j}\right)\right)^{-\alpha / n}\left(\sum_{k=j-2}^{\infty} \mid\left(\omega_{1}\left(B_{k}\right)\right)^{\alpha p / n}\left\|f \chi_{k}\right\|_{L^{q}}^{p}\right)^{1 / p} \\
\leq & C\left(\omega_{2}\left(B_{j}\right)\right)^{-1 / q}\left(\omega_{1}\left(B_{j}\right)\right)^{-\alpha / n}\|f\|_{\dot{K}_{q}^{\alpha, p}\left(\omega_{1}, \omega_{2}\right)} .
\end{aligned}
$$

Repeating the arguments used for the term $F_{1}$, we can also obtain

$$
F_{3} \leq C \dot{K}_{q}^{\alpha, p}\left(\omega_{1}, \omega_{2}\right) .
$$

Combining the above estimates for $F_{1}, F_{2}$ and $F_{3}$, and taking the supremum for all $\lambda>0$, the proof of Theorem 2 is finished.

\section{Proof of Theorem 3}

Let $f \in \dot{K}_{q}^{\alpha, p}\left(\omega_{1}, \omega_{2}\right)$, then

$$
\begin{aligned}
& \left\|\left[b, \mu_{\Omega}^{\rho}\right](f)\right\|_{\dot{K}_{q}^{\alpha, p}\left(\omega_{1}, \omega_{2}\right)}^{p} \\
\leq & C \sum_{j=-\infty}^{\infty}\left(\omega_{1}\left(B_{j}\right)\right)^{\frac{\alpha p}{n}}\left(\sum_{k=-\infty}^{j-2} \|\left(\left[b, \mu_{\Omega}^{\rho}\right]\left(f \chi_{k}\right) \chi_{j}(x) \|_{L^{q}\left(\omega_{2}\right)}\right)^{p}\right. \\
& +C \sum_{j=-\infty}^{\infty}\left(\omega_{1}\left(B_{j}\right)\right)^{\frac{\alpha p}{n}}\left(\sum_{k=j-1}^{j+1} \|\left(\left[b, \mu_{\Omega}^{\rho}\right]\left(f \chi_{k}\right) \chi_{j}(x) \|_{L^{q}\left(\omega_{2}\right)}\right)^{p}\right.
\end{aligned}
$$




$$
\begin{aligned}
& \text { PARAMETRIC MARCINKIEWICZ INTEGRALS ON WEIGHTED HERZ SPACES } \\
& +C \sum_{j=-\infty}^{\infty}\left(\omega_{1}\left(B_{j}\right)\right)^{\frac{\alpha p}{n}}\left(\sum_{k=j+2}^{\infty} \|\left(\left[b, \mu_{\Omega}^{\rho}\right]\left(f \chi_{k}\right) \chi_{j}(x) \|_{L^{q}\left(\omega_{2}\right)}\right)^{p}\right. \\
& =G_{1}+G_{2}+G_{3} .
\end{aligned}
$$

By the fact that $\left[b, \mu_{\Omega}^{\rho}\right]$ is a bounded operator on $L^{q}\left(\omega_{2}\right)$, we obtain

$G_{2} \leq C \sum_{j=-\infty}^{\infty}\left(\omega_{1}\left(B_{j}\right)\right)^{\frac{\alpha p}{n}}\left(\sum_{k=j-1}^{j+1}\left\|f \chi_{k}\right\|_{L^{q}\left(\omega_{2}\right)}\right)^{p} \leq C\|b\|_{*}^{p}\|f\|_{\dot{K}_{q}^{\alpha, p}\left(\omega_{1}, \omega_{2}\right)}^{p}$.

Obviously,

$$
\begin{aligned}
& \left\|\left[b, \mu_{\Omega}^{\rho}\right]\left(f \chi_{k}\right) \chi_{j}(x)\right\|_{L^{q}\left(\omega_{2}\right)} \\
\leq & C\left(\int_{C_{j}}\left|\left(b(x)-b_{B_{k}}\right) \mu_{\Omega}^{\rho}\left(f_{k}\right)(x)\right|^{q} \omega_{2}(x) d x\right)^{1 / q} \\
& +C\left(\int_{C_{j}}\left|\mu_{\Omega}^{\rho}\left(\left(b(\cdot)-b_{B_{k}}\right) f_{k}\right)(x)\right|^{q} \omega_{2}(x) d x\right)^{1 / q} \\
= & H_{1}+H_{2} .
\end{aligned}
$$

For the term $G_{1}$, since $j \geq k+2$, by (3.1) we have

$$
\begin{aligned}
H_{1}= & C\left(\int_{C_{j}}\left|\left(b(x)-b_{B_{k}}\right) \mu_{\Omega}^{\rho}\left(f_{k}\right)(x)\right|^{q} \omega_{2}(x) d x\right)^{1 / q} \\
\leq & C 2^{-j n}\left\|f \chi_{k}\right\|_{L^{1}}\left(\int_{B_{j}}\left|b(x)-b_{B_{k}}\right|^{q} \omega_{2}(x) d x\right)^{1 / q} \\
\leq & C 2^{-j n}\left\|f \chi_{k}\right\|_{L^{1}}\left(\left(\int_{B_{j}}\left|b(x)-b_{B_{j}, \omega_{2}}\right|^{q} \omega_{2}(x) d x\right)^{1 / q}\right. \\
& \left.+\left(\left|b_{B_{j}}-b_{B_{j}, \omega_{2}}\right|+\left|b_{B_{j}}-b_{B_{k}}\right|\right)\left(\int_{B_{j}} \omega_{2}(x) d x\right)^{1 / q}\right) .
\end{aligned}
$$

By Lemma 4 and the definition of $B M O\left(\mathbb{R}^{n}\right)$, we have

$$
\begin{gathered}
\left(\int_{B_{j}}\left|b(x)-b_{B_{j}, \omega_{2}}\right|^{q} \omega_{2}(x) d x\right)^{1 / q} \leq C\|b\|_{*}\left(\int_{B_{j}} \omega_{2}(x) d x\right)^{1 / q}, \\
\left|b_{B_{j}}-b_{B_{j}, \omega_{2}}\right| \leq C\|b\|_{*},
\end{gathered}
$$

and

$$
\left|b_{B_{j}}-b_{B_{k}}\right| \leq C(j-k)\|b\|_{*} .
$$


From (3.2),(3.3) and (5.1)-(5.3), we get

$$
\begin{aligned}
H_{1} & \leq C\|b\|_{*}\left\|f \chi_{k}\right\|_{L^{q}\left(\omega_{2}\right)}(j-k) 2^{-j n}\left(\int_{B_{j}} \omega_{2}(x) d x\right)^{1 / q}\left(\int_{B_{k}} \omega_{2}(x)^{1-q^{\prime}} d x\right)^{1 / q^{\prime}} \\
& \leq C\|b\|_{\star}\left\|f \chi_{k}\right\|_{L^{q}\left(\omega_{2}\right)}(j-k) 2^{(j-k) n\left(q_{2} / q-1\right)}
\end{aligned}
$$

for $j \geq k+2$.

Similar to the estimate of (3.1),

$$
\left|\mu_{\Omega}^{\rho}\left(\left(b(\cdot)-b_{B_{k}}\right) f_{k}\right)(x)\right| \leq C 2^{-j n}\left(\int_{C_{k}}\left|b(z)-b_{B_{k}}\right||f(z)| d z\right) .
$$

So

$$
\begin{gathered}
\left(\int_{C_{j}}\left|\mu_{\Omega}^{\rho}\left(\left(b(\cdot)-b_{B_{k}}\right) f_{k}\right)(x)\right|^{q} \omega_{2}(x) d x\right)^{1 / q} \\
\leq C 2^{-j n}\left(\omega_{2}\left(B_{j}\right)\right)^{1 / q}\left(\int_{C_{k}}\left|b(z)-b_{B_{k}}\right||f(z)| d z\right) .
\end{gathered}
$$

Since $\omega_{2} \in A_{q_{2}}$, by Lemma 1 we know that $\omega_{2}^{1-q_{2}^{\prime}} \in A_{q_{2}^{\prime}}$. Therefore by Lemma 4 we have

$$
\left(\int_{B_{k}}\left|b(x)-b_{B_{k}}\right|^{q^{\prime}} \omega_{2}(x)^{1-q^{\prime}} d x\right)^{1 / q^{\prime}} \leq C\|b\|_{*}\left(\int_{B_{k}} \omega_{2}(x)^{1-q^{\prime}} d x\right)^{1 / q^{\prime}} .
$$

Using Hölder's inequality , (3.3) and (5.4) we get

$$
\begin{aligned}
H_{2} & \leq C 2^{-j n} \int_{B_{k}}\left|b(y)-b_{B_{k}}\right|\left|f \chi_{k}(y)\right| d y\left(\int_{B_{j}} \omega_{2}(x) d x\right)^{1 / q} \\
& \leq C 2^{-j n}\left(\int_{B_{k}}\left|b(y)-b_{B_{k}}\right|^{q^{\prime}} \omega_{2}(y)^{1-q^{\prime}} d y\right)^{1 / q^{\prime}}\left\|f \chi_{k}\right\|_{L^{q}\left(\omega_{2}\right)}\left(\int_{B_{j}} \omega_{2}(x) d x\right)^{1 / q} \\
& \leq C\|b\|_{*}\left\|f \chi_{k}\right\|_{L^{q}\left(\omega_{2}\right)^{2}} 2^{-j n}\left(\int_{B_{k}} \omega_{2}(x)^{1-q^{\prime}} d x\right)^{1 / q^{\prime}}\left(\int_{B_{j}} \omega_{2}(x) d x\right)^{1 / q} \\
& \leq C\|b\|_{*}\left\|f \chi_{k}\right\|_{L^{q}\left(\omega_{2}\right)} 2^{(j-k) n\left(q_{2} / q-1\right)} .
\end{aligned}
$$

Summarizing the above estimates, we have that for $j \geq k+2$,

$$
\left\|\left[b, \mu_{\Omega}^{\rho}\right]\left(f \chi_{k}\right) \chi_{j}(x)\right\|_{L^{q}\left(\omega_{2}\right)} \leq C\|b\|_{*}\left\|f \chi_{k}\right\|_{L^{q}\left(\omega_{2}\right)}(j-k) 2^{(j-k) n\left(q_{2} / q-1\right)} .
$$

Using (5.5) and repeating the estimation process of $E_{1}$, we obtain

$$
G_{1} \leq C\|b\|_{*}^{p}\|f\|_{\dot{K}_{q}^{\alpha, p}\left(\omega_{1}, \omega_{2}\right)}^{p}
$$

for $0<p<\infty$. 
Finally, let us estimate $G_{3}$. Since $j \leq k-2$, by (3.4) we have

$$
\begin{aligned}
H_{1}= & C\left(\int_{C_{j}}\left|\left(b(x)-b_{B_{k}}\right) \mu_{\Omega}^{\rho}\left(f_{k}\right)(x)\right|^{q} \omega_{2}(x) d x\right)^{1 / q} \\
& \leq C 2^{-k n}\left\|f \chi_{k}\right\|_{L^{1}}\left(\int_{B_{j}}\left|b(x)-b_{B_{k}}\right|^{q} \omega_{2}(x) d x\right)^{1 / q} .
\end{aligned}
$$

From (3.2),(5.1)-(5.3) and (3.6), we get

$$
\begin{aligned}
H_{1} & \leq C\|b\|_{*}\left\|f \chi_{k}\right\|_{L^{q}\left(\omega_{2}\right)}(j-k) 2^{-j n}\left(\int_{B_{j}} \omega_{2}(x) d x\right)^{1 / q}\left(\int_{B_{k}} \omega_{2}(x)^{1-q^{\prime}} d x\right)^{1 / q^{\prime}} \\
& \leq C\|b\|_{\star}\left\|f \chi_{k}\right\|_{L^{q}\left(\omega_{2}\right)}(j-k) 2^{(j-k) \delta_{2} n / q}
\end{aligned}
$$

for $j \leq k-2$.

Similar to the estimate of (3.4),

$$
\left|\mu_{\Omega}^{\rho}\left(\left(b(\cdot)-b_{B_{k}}\right) f_{k}\right)(x)\right| \leq C 2^{-k n}\left(\int_{C_{k}}\left|b(z)-b_{B_{k}}\right||f(z)| d z\right) .
$$

So

$$
\begin{aligned}
& \left(\int_{C_{j}}\left|\mu_{\Omega}^{\rho}\left(\left(b(\cdot)-b_{B_{k}}\right) f_{k}\right)(x)\right|^{q} \omega_{2}(x) d x\right)^{1 / q} \\
\leq & C 2^{-k n}\left(\omega_{2}\left(B_{j}\right)\right)^{1 / q}\left(\int_{C_{k}}\left|b(z)-b_{B_{k}}\right||f(z)| d z\right) .
\end{aligned}
$$

Using Hölder's inequality, (5.4) and (3.6),

$$
\begin{aligned}
H_{2} & \leq C 2^{-k n} \int_{B_{k}}\left|b(y)-b_{B_{k}}\right|\left|f \chi_{k}(y)\right| d y\left(\int_{B_{j}} \omega_{2}(x) d x\right)^{1 / q} \\
& \leq C 2^{-k n}\left(\int_{B_{k}}\left|b(y)-b_{B_{k}}\right|^{q^{\prime}} \omega_{2}(y)^{1-q^{\prime}} d y\right)^{1 / q^{\prime}}\left\|f \chi_{k}\right\|_{L^{q}\left(\omega_{2}\right)}\left(\int_{B_{j}} \omega_{2}(x) d x\right)^{1 / q} \\
& \leq C\|b\|_{*}\left\|f \chi_{k}\right\|_{L^{q}\left(\omega_{2}\right)} 2^{-k n}\left(\int_{B_{k}} \omega_{2}(x)^{1-q^{\prime}} d x\right)^{1 / q^{\prime}}\left(\int_{B_{j}} \omega_{2}(x) d x\right)^{1 / q} \\
& \leq C\|b\|_{*}\left\|f \chi_{k}\right\|_{L^{q}\left(\omega_{2}\right)} 2^{(j-k) \delta_{2} n / q} .
\end{aligned}
$$

Thus

$$
\left\|\left[b, \mu_{\Omega}^{\rho}\right]\left(f \chi_{k}\right) \chi_{j}(x)\right\|_{L^{q}\left(\omega_{2}\right)} \leq C\|b\|_{*}\left\|f \chi_{k}\right\|_{L^{q}\left(\omega_{2}\right)}(k-j) 2^{(j-k) \delta_{2} n / q}
$$

for $j \geq k-2$. Repeating the estimation process of $E_{3}$, we obtain

$$
G_{3} \leq C\|b\|_{*}^{p}\|f\|_{\dot{K}_{q}^{\alpha, p}\left(\omega_{1}, \omega_{2}\right)}^{p}
$$

for $0<p<\infty$. 

3.

Summing up the estimates of $G_{1}, G_{2}$ and $G_{3}$, it completes the proof of Theorem

\section{ACKNOWLEDGEMENT}

The author is very grateful to the anonymous referees and the editor for their insightful comments and suggestions.

\section{REFERENCES}

[1] A. Al-Salman, "On the $l^{2}$-boundedness of parametric marcinkiewicz integral operator," J. Math. Anal. Appl., vol. 375, no. 2, pp. 745-752, 2011, doi: 10.1016/j.jmaa.2010.09.062.

[2] S. Aliev and V. Guliev, "Boundedness of the parametric marcinkiewicz integral operator and its commutators on generalized morrey spaces," Georgian Math. J., vol. 19, no. 2, pp. 195-208, 2012.

[3] Q. Fang and X. Shi, "Estimates for parametric marcinkiewicz integrals in bmo and campanato spaces," Appl. Math. J. Chinese Univ. Ser. B, vol. 26, no. 2, pp. 230-252, 2011, doi: 10.1007/s11766-011-2685-7.

[4] L. Grafakos, "Classical and modern fourier analysis," Pearson Education, Inc., Upper Saddle River, NJ, 2004.

[5] Y. Guo and Y. Jiang, "Weighted herz space and regularity results," J. Funct. Spaces Appl., no. 283730 , p. 13, 2012.

[6] L. Hörmander, "Estimates for translation invariant operators in $l^{p}$ spaces," Acta Math., vol. 104, pp. 93-140, 1960, doi: 10.1007/BF02547187.

[7] F. John and L. Nirenberg, "On functions of bounded mean oscillation," Comm. Pure Appl. Math., vol. 14, pp. 415-426, 1961, doi: 10.1002/cpa.3160140317.

[8] Y. Komori and K. Matsuoka, "Boundedness of several operators on weighted herz spaces," $J$. Funct. Spaces Appl., vol. 7, no. 1, pp. 1-12, 2009, doi: 10.1155/2009/739134.

[9] S. Lu and D. Yang, "The decomposition of weighted herz space on $\mathbb{R}^{n}$ and its applications," Sci. China Ser. A., vol. 38, no. 2, pp. 147-158, 1995.

[10] B. Muckenhoupt, "Weighted norm inequalities for the hardy maximal function," Trans. Amer. Math. Soc., vol. 165, pp. 207-226, 1972, doi: 10.1090/S0002-9947-1972-0293384-6.

[11] B. Muckenhoupt and R. Wheeden, "Weighted bounded mean oscillation and the hilbert transform," Studia Math., vol. 54, no. 3, pp. 221-237, 1975/76.

[12] Z. F. S Gong and B. Ma, "Weighted multilinear hardy operators on herz type spaces," The Scientific World Journal, no. 420408, p. 10, 2014.

[13] K. Y. S. Lu and D. Yang, "Boundedness of some sublinear operators in weighted herz-type spaces," Kodai Math. J., vol. 23, no. 3, pp. 391-410, 2000, doi: 10.2996/kmj/1138044267.

[14] X. Shi and Y. Jiang, "Weighted boundedness of parametric marcinkiewicz integral and higher order commutator," Anal. Theory Appl., vol. 25, no. 1, pp. 25-39, 2009, doi: 10.1007/s10496009-0025-z.

[15] E. Stein, "On the functions of littlewood-paley," Lusin and Marcinkiewicz, Trans. Amer. Math. Soc., vol. 88, pp. 430-466, 1958, doi: 10.1090/S0002-9947-1958-0112932-2.

[16] A. Torchinsky and S. Wang, "A note on the marcinkiewicz integral," Colloq. Math., vol. 60/61, no. 2, pp. 235-243, 1990.

[17] H. Wang, "The boundedness of intrinsic square functions on the weighted herz spaces," J. Funct. Spaces, no. 274521, p. 14, 2014.

[18] Q. X. Y. Ding and K. Yabuta, "Ba remark to the $l^{2}$ boundedness of parametric marcinkiewicz integral," J. Math. Anal. Appl., vol. 387, no. 2, pp. 691-697, 2012, doi: 10.1016/j.jmaa.2011.09.020. 
[19] Y. H. Y. Hu and Y. Wang, "The commutators of fractional integrals on generalized herz spaces," J. Funct. Spaces, no. 428493, p. 6, 2014.

Authors' addresses

Yue Hu

Henan Polytechnic University, College of Mathematics and Informatics, 454003, Jiaozuo, Henan, China

E-mail address: huu3y3@email.com

Yueshan Wang

Jiaozuo University, Department of Mathematics, 454003, Jiaozuo, Henan, China

E-mail address: wangys1962@163.com 